\title{
Tidal pull of the Earth strips the proto-Moon of its volatiles
}

\author{
SEBASTIEN CHARNOZ ${ }^{1}$, PAOLO A. SOSSI ${ }^{2}$, YUEH-NING \\ LEE $^{3}$, JULIEN SIEBERT ${ }^{4}$, RYUKI HYODO ${ }^{5}$, LAETITIA \\ ALLIBERT $^{6}$, MAYLIS LANDEAU $^{4}$, APURVA OZA $^{7}$ AND \\ FRÉDÉRIC MOYNIER ${ }^{8}$ \\ ${ }^{1}$ Université de Paris / IPGP/ CNRS / UMR 7154 \\ ${ }^{2}$ ETH Zürich \\ ${ }^{3}$ Department of Earth Sciences, National Taiwan Normal \\ University \\ ${ }^{4}$ Institut de Physique du Globe de Paris \\ ${ }^{5}$ Earth Life Science Institute/Tokyo Institute of Technology \\ ${ }^{6}$ Museum für Naturkunde Leibniz-Institut für Evolutions- und \\ Biodiversitätsforschung \\ ${ }^{7}$ Physikalisches Institut, University of Bern \\ ${ }^{8}$ Université de Paris, Institut de physique du globe de Paris \\ Presenting Author: charnoz@ipgp.fr
}

Prevailing models for the formation of the Moon invoke a giant impact between a planetary embryo and the proto-Earth. Despite similarities in the isotopic and chemical abundances of refractory elements compared to Earth's mantle, the Moon is depleted in volatiles. Current models favour devolatilisation via incomplete condensation of the proto- Moon in an Earth-Moon debris-disk. However the physics of this protolunar disk is poorly understood and thermal escape of gas is inhibited by the Earth's strong gravitational field. Here we investigate a simple process, wherein the Earth's tidal pull promotes intense hydrodynamic escape from the surface of a molten proto-Moon assembling at 36 Earth radii. Such tidally-driven atmospheric escape persisting for less than $1 \mathrm{Kyr}$ at temperatures $\sim 1600-1700 \mathrm{~K}$ reproduces the measured lunar depletion in $\mathrm{K}$ and $\mathrm{Na}$, assuming the escape starts just above the surface. These results are in accord with timescales for the rapid solidification of a plagioclase lid at the surface of a lunar magma ocean (Elkins-Tanton et al. 2011). We find that Hydrodynamic escape, both in an adiabatic or isothermal regime, with or without condensation, induces advective transport of gas away from the lunar surface, causing a decrease in the partial pressures of gas species (Ps) with respect to their equilibrium values (Psat). The observed enrichment in heavy stable isotopes of $\mathrm{Zn}$ and $\mathrm{K}$.constrain Ps/Psat $>0.99$, favouring a scenario in which volatile loss occurred at low Hydrodynamic wind velocities and thus low temperatures. We conclude that tidally-driven atmospheric escape is an unavoidable consequence of the Moon's assembly under the gravitational influence of the Earth, and provides new pathways toward understanding lunar formation. 